\title{
Intrinsic alignment-lensing interference as a contaminant of cosmic shear
}

\author{
Christopher M. Hirata ${ }^{1, *}$ and Uroš Seljak ${ }^{1, \dagger}$ \\ ${ }^{1}$ Dept. of Physics, Jadwin Hall, Princeton University, Princeton, New Jersey 08544, USA
}

(Received 15 June 2004; published 21 September 2004)

\begin{abstract}
Cosmic shear surveys have great promise as tools for precision cosmology, but can be subject to systematic errors including intrinsic ellipticity correlations of the source galaxies. The intrinsic alignments are believed to be small for deep surveys, but this is based on intrinsic and lensing distortions being uncorrelated. Here we show that the gravitational lensing shear and intrinsic shear need not be independent: correlations between the tidal field and the intrinsic shear cause the intrinsic shear of nearby galaxies to be correlated with the gravitational shear acting on more distant galaxies. We estimate the magnitude of this effect for two simple intrinsic-alignment models: one in which the galaxy ellipticity is linearly related to the tidal field, and one in which it is quadratic in the tidal field as suggested by tidal torque theory. The first model predicts a gravitational-intrinsic $(G I)$ correlation that can be much greater than the intrinsic-intrinsic (II) correlation for broad redshift distributions, and that remains when galaxies pairs at similar redshifts are rejected. The second model, in its simplest form, predicts no gravitational-intrinsic correlation. In the first model, and assuming a normalization consistent with recently claimed detections of intrinsic correlations, we find that the $G I$ correlation term can exceed the usual $I I$ term by $>1$ order of magnitude and the intrinsic correlation induced $B$-mode by 2 orders of magnitude. These interference effects can suppress the lensing power spectrum for a single broad redshift bin by of order $\sim 10 \%$ at $z_{s}=1$ and $\sim 30 \%$ at $z_{s}=0.5$. We conclude that, depending on the intrinsic-alignment model, the GI correlation may be the dominant contaminant of the lensing signal and can even affect cross spectra between widely separated bins. We describe two ways to constrain this effect, one based on density-shear correlations and one based on scaling of the cross correlation tomography signal with redshift.
\end{abstract}

DOI: $10.1103 /$ PhysRevD.70.063526

PACS numbers: $98.80 . \mathrm{Es}, 98.62 . \mathrm{Gq}, 98.62 . \mathrm{Sb}$

\section{INTRODUCTION}

Weak gravitational lensing has attracted considerable interest recently as a means of measuring the density perturbations in the universe at low redshifts because it is directly sensitive to the matter distribution. It thus avoids the astrophysical complications involved in other means of measuring the matter distribution such as galaxy clustering. Recent first detections of the cosmic shear autopower spectrum [1-8] have stimulated proposals for more ambitious projects in the future, such as CFHTLS [9], Pan-STARRS [10], SNAP[11], and LSST [12], which aim for percent-level precision. Comparison of the shear power spectra at different redshifts and/or to the primordial fluctuation amplitude derived from cosmic microwave background (CMB) anisotropy measurements can constrain the cosmological growth factor and hence parameters such as the neutrino mass, amplitude of fluctuations $\sigma_{8}$, and dark energy equation of state [13-18].

While the underlying physics of weak lensing is "clean," the shear measurements are subject to possible systematic errors, including incomplete correction for seeing and optical distortions, selection effects, and noise-rectification biases [19-24], and their cosmological interpretation relies on accurate knowledge of the redshift distribution of the source galaxies. Another possible

\footnotetext{
*Electronic address: chirata@princeton.edu
}

†Electronic address: useljak@princeton.edu systematic error is intrinsic (i.e. not lensing-induced) correlations among the ellipticities of neighboring source galaxies [25-34], which could arise if the galaxy ellipticities are affected by large-scale tidal fields. This systematic error is particularly worrisome because it lies outside the control of the observer, and is dependent upon the poorly understood physics of galaxy formation. One frequently proposed method to reduce this contamination is to assign photometric redshifts to the source galaxies, and then to down-weight or ignore pairs of galaxies at similar redshifts when computing the shear correlation function or power spectrum [35-38]; see Ref. [39] for an implementation. The idea is that the pairs of galaxies widely separated in redshift should have independent intrinsic ellipticities, but should have correlated gravitational shears induced by structures between the observer and the more nearby source galaxy.

While cross correlation of different redshift bins is expected to remove spurious power due to the intrinsic alignment autocorrelation, there is another more subtle effect by which even the cross correlations can be contaminated from intrinsic alignments. If the ellipticities of galaxies are correlated with the tidal quadrupole field in which the galaxies form, then the intrinsic ellipticity of a nearby source galaxy will be correlated with the lensing shear acting on a more distant source galaxy. This leads to a nonzero cross correlation between the intrinsic ellipticity and the gravitational lensing shear. Cross correlations between the shear measurements at widely different red- 
shifts are actually more contaminated by this effect than shear autocorrelations computed using only galaxies in a narrow redshift slice because the radial separation is necessary in order for the tidal field around the nearby galaxy to lens the more distant galaxy. This intrinsic lensing correlation will also affect attempts to cross correlate cosmic shear surveys of galaxies with lensing of the CMB [17,40,41] because the CMB is also lensed by the tidal field surrounding the source galaxies.

This paper is organized as follows. In Sec. II, we formally express the $E$ - and $B$-mode shear power spectra in terms of the background cosmology, power spectra of matter density and intrinsic shear, and matter-shear cross spectrum. In Sec. III we consider two crude models of intrinsic alignments and calculate their predicted contribution to the shear power spectrum. We discuss methods to assess and/or remove the contamination in Sec. IV, and we conclude in Sec. V.

\section{SHEAR POWER SPECTRA}

Before developing the formalism, let us describe a simple example of the effect, shown in Fig. 1. The tidal field may lead to a stretching of the galaxy shape in the direction of the tidal field. Gravitational shearing of a background source leads to stretching of the galaxy in perpendicular direction. As a result, the lensing effect will be partially cancelled by the intrinsic alignment effect and the two effects are coherent, as they depend on the same underlying density field.

Weak gravitational lensing by large-scale structures is detectable through its shearing of distant "source" galaxies. To lowest order, the shear of a galaxy $i$ can be broken down into a gravitational and an intrinsic shear contribution $\gamma_{i}=\gamma_{i}^{G}+\gamma_{i}^{I}$. The gravitational shear is well known and is equal to

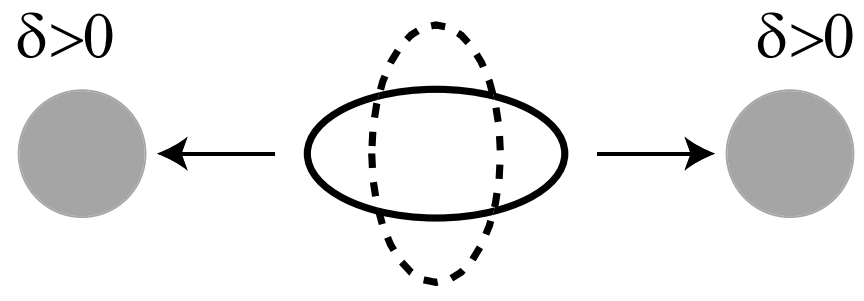

FIG. 1. The effect of the density-intrinsic shear correlation on the shear power spectrum. Density fluctuations in the nearby plane (gray masses) induce a tidal field (arrows). A source galaxy in a more distant plane (dashed ellipse) is gravitationally sheared tangentially to these masses. If the intrinsic shears of galaxies in the nearby plane (solid ellipse) are aligned with the stretching axis of the tidal field, then this results in an anticorrelation between the shears of galaxies at different redshifts, i.e. $C_{\ell}^{E E, G I}<0$. (The opposite case, $C_{\ell}^{E E, G I}>0$, results if galaxies are preferentially aligned with the compressing axis of the tidal field.)
$\left(\gamma_{i+}^{G}, \gamma_{i \times}^{G}\right)=\partial^{-2} \int_{0}^{\infty} W\left(\chi, \chi_{i}\right)\left(\partial_{x}^{2}-\partial_{y}^{2}, 2 \partial_{x} \partial_{y}\right) \delta\left(\chi \hat{\mathbf{n}}_{i}\right) d \chi$,

where the $\partial$ derivatives are takes with respect to angular position (i.e. have units of radians -1 ), $\partial^{-2}$ is the associated inverse Laplacian, $\hat{\mathbf{n}}_{i}$ is the angular position of galaxy $i, \delta(\chi \hat{\mathbf{n}})$ is the fractional density perturbation at distance $\chi$ in direction $\hat{\mathbf{n}}$, and the lensing window function is

$$
W\left(\chi, \chi_{i}\right)=\frac{3}{2} \Omega_{m} H_{0}^{2}(1+z) \sin _{K}^{2} \chi\left(\cot _{K} \chi-\cot _{K} \chi_{i}\right)
$$

for $\chi<\chi_{i}$ and 0 otherwise. Here $\sin _{K}$ and $\cot _{K}$ are the modified trigonometric functions, i.e.

$$
\sin _{K} \chi= \begin{cases}K^{-1 / 2} \sin \left(K^{1 / 2} \chi\right) & K>0 \\ \chi & K=0 \\ |K|^{-1 / 2} \sinh \left(|K|^{1 / 2} \chi\right) & K<0\end{cases}
$$

$\cot _{K} \chi=\frac{d}{d \chi} \ln _{\sin } \chi$, and $K$ is the spatial curvature of the universe.

Now we imagine that a set of source galaxies in redshift slice $\alpha$ with comoving distance distribution $f_{\alpha}(\chi)$ are observed. The gravitational contribution to a shear Fourier mode is

$$
\gamma_{l}^{G}(\alpha)=\left(\cos 2 \phi_{l}, \sin 2 \phi_{l}\right) \int_{0}^{\infty} W_{\alpha}(\chi) \delta_{l}(\chi) d \chi,
$$

where the integrated window function is

$$
W_{\alpha}(\chi)=\int_{0}^{\infty} f_{\alpha}\left(\chi^{\prime}\right) W\left(\chi, \chi^{\prime}\right) d \chi^{\prime}
$$

The intrinsic alignment contribution at a point is given by

$$
\gamma^{I}(\hat{\mathbf{n}}, \alpha)=\int_{0}^{\infty} f_{\alpha}(\chi) \tilde{\gamma}^{I}(\chi \hat{\mathbf{n}}) d \chi,
$$

where the density-weighted intrinsic shear $\tilde{\gamma}^{I}=$ $\left(1+\delta_{g}\right) \gamma^{I}$ is computed from the fractional overdensity of galaxies $\delta_{g}$ and average intrinsic shear of galaxies $\gamma^{I}$. A density weighting is technically necessary in Eq. (5), which makes $W_{\alpha}(\chi)$ slightly dependent on angular position. On subarcminute scales where the fluctuations in $\delta_{g}$ are large this results in production of $B$-modes in the lensing shear [42]; on larger scales the effect is unimportant. In contrast, intrinsically aligned pairs of galaxies tend to be close to each other where $\delta_{g} \geq 1$, and hence the factor of $1+\delta_{g}$ in Eq. (6) cannot be safely neglected except on very large scales.

The $E$-mode shear cross spectrum between two redshift slices can be broken down into gravitational lensing $(G G)$, intrinsic alignment $(I I)$, and interference $(G I)$ terms 


$$
C_{\ell}^{E E}(\alpha \beta)=C_{\ell}^{E E, G G}(\alpha \beta)+C_{\ell}^{E E, I I}(\alpha \beta)+C_{\ell}^{E E, G I}(\alpha \beta) .
$$

(The $B$-mode shear cross spectrum is similar, but contains only an $I I$ term since there is no gravitational contribution to the $B$-mode shear.) The gravitational lensing contribution to the shear power spectrum can be obtained on small scales $(\ell \gg 1)$ by Limber integration [43], which computes the power spectrum of a radially projected quantity. In this case we integrate Eq. (4) to get

$$
C_{\ell}^{E E, G G}(\alpha \beta)=\int_{0}^{\infty} \frac{W_{\alpha}(\chi) W_{\beta}(\chi)}{\sin _{K}^{2} \chi} P_{\delta}\left(k=\frac{\ell}{\sin _{K} \chi} ; \chi\right) d \chi .
$$

The intrinsic alignment contribution is most easily computed by Limber integration of Eq. (6)

$$
C_{\ell}^{E E, I I}(\alpha \beta)=\int_{0}^{\infty} \frac{f_{\alpha}(\chi) f_{\beta}(\chi)}{\sin _{K}^{2} \chi} P_{\tilde{\gamma}^{I}}^{E E}\left(k=\frac{\ell}{\sin _{K} \chi} ; \chi\right) d \chi,
$$

where the projected power spectrum of the intrinsic alignments is

$$
\begin{aligned}
P_{\tilde{\gamma}^{I}}^{E E}(k ; \chi)= & \pi \iint_{\int}\left\{\left\langle\tilde{\gamma}_{+}^{I}(0) \tilde{\gamma}_{+}^{I}(x)\right\rangle\left[J_{0}\left(k x_{\perp}\right)+J_{4}\left(k x_{\perp}\right)\right]\right. \\
& \left.+\left\langle\tilde{\gamma}_{\times}^{I}(0) \tilde{\gamma}_{\times}^{I}(x)\right\rangle\left[J_{0}\left(k x_{\perp}\right)-J_{4}\left(k x_{\perp}\right)\right]\right\} \\
& \times x_{\perp} d x_{\perp} d x_{\|},
\end{aligned}
$$

where the \langle\rangle are two-point correlation functions of $\tilde{\gamma}$ with two points separated by comoving distance $x_{\|}$in the radial direction and $x_{\perp}$ in the perpendicular direction. The + and $\times$ components of the shear are measured along the direction of separation $\mathbf{x}_{\perp}$ and at 45 degrees to this direction, respectively. Note that in general the two-point correlation functions can depend on $x_{\perp}$ and $x_{\|}$ independently (i.e., they are not just functions of the separation $\sqrt{x_{\perp}^{2}+x_{\|}^{2}}$ ) since the shear depends on viewing geometry. To get the $B$-mode power, switch the + and $\times$ labels in Eq. (10).

Given models for intrinsic alignment and galaxy clustering, Eqs. (9) and (10) are sufficient to compute the pure intrinsic alignment contribution to the shear cross spectrum. However, note that as a second-order statistic, Eq. (7) also contains interference terms due to correlation of the gravitational shear with intrinsic alignments. These have normally been ignored because the gravitational shear acting on a particular galaxy is determined by the integrated tidal quadrupole along the line of sight, whereas the intrinsic shear is expected to be determined by tidal fields in the vicinity of the galaxy. But in cosmic shear studies, we correlate the shears of two galaxies whose redshifts may be different. In this case, it is plausible that the more nearby galaxy is intrinsically aligned by a quadrupolar tidal field that also lenses the more distant galaxy. Therefore we must consider the interference terms. These are given by

$$
\begin{aligned}
C_{\ell}^{E E, G I}(\alpha \beta)= & \int_{0}^{\infty} \frac{W_{\alpha}(\chi) f_{\beta}(\chi)+W_{\beta}(\chi) f_{\alpha}(\chi)}{\sin _{K}^{2} \chi} \\
& \times P_{\delta, \tilde{\gamma}^{I}}\left(\frac{\ell}{\sin _{K} \chi}\right) d \chi,
\end{aligned}
$$

where the 3-dimensional cross spectrum $P_{\delta, \tilde{\gamma}^{I}}$ is related to the density-shear correlation function via

$$
P_{\delta, \tilde{\gamma}^{\prime}}(k ; \chi)=-2 \pi \iint\left\langle\delta(0) \tilde{\gamma}_{+}^{I}(x)\right\rangle J_{2}\left(k x_{\perp}\right) x_{\perp} d x_{\perp} d x_{\|} .
$$

\section{MODELS}

We now compute the predicted level of contamination of weak lensing surveys from the "interference" terms, Eq. (11). We consider two cases: first the case of galaxies whose mean ellipticities are linear functions of the underlying tidal field; and second, the case of galaxies whose mean ellipticities are quadratic functions of the tidal field. In all of our numerical results below, we have taken the bias to be $b_{g}=1$, used the best-fit scale-invariant $\left(n_{s}=\right.$ 1) flat $\Lambda$ CDM model of Ref. [44] $\left(\sigma_{8}=0.966, \Omega_{b}=\right.$ $0.0475, \Omega_{m}=0.293, H_{0}=70.8 \mathrm{~km} / \mathrm{s} / \mathrm{Mpc}$ ), and used the adiabatic $\mathrm{CDM}+$ baryon transfer function of Ref. [45]. The gravitational-gravitational shear power spectra include the nonlinear correction of Ref. [46].

The first (linear) model has usually been used for elliptical galaxies (since gravitational collapse in a general tidal field is expected to result in a triaxial halo aligned with the principal axes of the tidal field) and the second (quadratic) model for spirals (since the orientation of these is believed to be determined by angular momentum acquired during gravitational collapse, which requires one tidal quadrupole to supply the torque and another tidal quadrupole to be torqued). The models presented here are "toy" models in the sense that their theoretical motivation does not apply to highly nonlinear scales, and even on larger scales there is little observational basis for models of intrinsic galaxy ellipticity alignments. An alternative to analytic models is to estimate intrinsic alignments by calculating the ellipticities or angular momenta of dark matter haloes in $N$-body simulations [25-27,32], however one should keep in mind that there can be misalignment between the dark matter halo and the galaxy it contains [47], and so intrinsic alignment results based on $N$-body simulations are not definitive. In principle it would be possible to derive predictions for intrinsic correlations from a halo model, however this would require adding a prescription for the galaxy alignment to the current halo models. Such a prescription would likely have to come from an analytic 
or $N$-body model. Undoubtedly there is much room for improvement in both the theory - which must ultimately address the complications of galaxy formation, mergers, etc.-and the observations, which will be necessary to measure or constrain the intrinsic alignment signal at levels suitable for precision cosmology.

\section{A. Linear alignment model}

A simple model for the ellipticities of elliptical galaxies was proposed by [30]. The intrinsic shear of the galaxy is assumed to follow the linear relation

$$
\gamma^{I}=-\frac{C_{1}}{4 \pi G}\left(\nabla_{x}^{2}-\nabla_{y}^{2}, 2 \nabla_{x} \nabla_{y}\right) S\left[\Psi_{P}\right]
$$

where $\Psi_{P}$ is the Newtonian potential at the time of galaxy formation (assumed to be during matter domination), $S$ is a smoothing filter that cuts off fluctuations on galactic scales, and $\nabla$ is a comoving derivative (as opposed to $\partial$ which is a 2-dimensional derivative on the unit sphere with units of radians -1 ). We have taken $S$ to be a simple cutoff in Fourier space at $k_{\text {cutoff }}=1 h / \mathrm{Mpc}$. Here $C_{1}$ is a normalization constant (note that $C_{1}>0$ if the galaxy is aligned along the "stretching" axis of the tidal field). The original motivation for Eq. (13) was the assumption that galaxies are homologous with their haloes, and that the halo ellipticity is perturbed by the local tidal field produced by large-scale structure [30]. On sufficiently large scales, this relation can also be motivated by arguments analogous to linear biasing theory for galaxies [48]: the large-scale correlations in the intrinsic shear field must be determined by the large-scale potential fluctuations; if these large-scale potential fluctuations are sufficiently small, then the intrinsic shear field should be a linear and local function of the potential $\Psi_{P}$ (which is $\propto \Psi$ in the linear regime); the only linear, local functions of $\Psi_{P}$ with quadrupole symmetry are Eq. (13) and derivatives thereof; and on large scales we expect higher-derivative terms such as $\nabla^{2}\left(\nabla_{x}^{2}-\nabla_{y}^{2}, 2 \nabla_{x} \nabla_{y}\right) S\left[\Psi_{P}\right]$ to be negligible.

The primordial potential is related to the linear density field via

$$
\Psi_{P}(\mathbf{k})=-4 \pi G \frac{\bar{\rho}(z)}{\bar{D}(z)} k^{-2} \delta_{\operatorname{lin}}(\mathbf{k})
$$

where $\bar{\rho}(z)$ is the mean density of the universe, $\bar{D}(z) \propto$ $(1+z) D(z)$ is the rescaled growth factor normalized to unity during matter domination, and $G$ is the Newtonian gravitational constant. On linear scales, we have $\delta_{g}=$ $b_{g} \delta_{\text {lin }}, \delta=\delta_{\text {lin }}$, and hence the weighted intrinsic shear is

$$
\begin{aligned}
& \tilde{\gamma}(\mathbf{k})=\frac{C_{1} \bar{\rho}}{\bar{D}} \int \frac{\left(k_{2 x}^{2}-k_{2 y}^{2}, 2 k_{2 x} k_{2 y}\right)}{k_{2}^{2}} \delta_{\operatorname{lin}}\left(\mathbf{k}_{2}\right)\left[\delta^{(3)}\left(\mathbf{k}_{1}\right)\right. \\
& \left.+\frac{b_{g}}{(2 \pi)^{3}} \delta_{\operatorname{lin}}\left(\mathbf{k}_{1}\right)\right] d^{3} \mathbf{k}_{1} \text {, }
\end{aligned}
$$

where $\mathbf{k}_{2} \equiv \mathbf{k}-\mathbf{k}_{1}$ and we have chosen the wave vector $\mathbf{k}$ to lie on the $x$-axis. (We are only interested in modes with $\mathbf{k}$ perpendicular to the line of sight.) The power spectrum of $\tilde{\gamma}$ is

$$
\begin{aligned}
P_{\tilde{\gamma}^{I}}^{E E}(k)= & \frac{C_{1}^{2} \bar{\rho}^{2}}{\bar{D}^{2}}\left\{P_{\delta}^{\operatorname{lin}}(k)+b_{g}^{2} \int\left[f_{E}\left(\mathbf{k}_{2}\right)+f_{E}\left(\mathbf{k}_{1}\right)\right]\right. \\
& \left.\times f_{E}\left(\mathbf{k}_{2}\right) \frac{P_{\delta}^{\operatorname{lin}}\left(k_{1}\right) P_{\delta}^{\operatorname{lin}}\left(k_{2}\right)}{(2 \pi)^{3}} d^{3} \mathbf{k}_{1}\right\}
\end{aligned}
$$

where $f_{E}(\mathbf{w})=\left(w_{x}^{2}-w_{y}^{2}\right) / w^{2}$. To get the $B$-mode power spectrum, we replace $f_{E}$ with $f_{B}(\mathbf{w})=2 w_{x} w_{y} / w^{2}$

$$
\begin{aligned}
P_{\tilde{\gamma}^{I}}^{B B}(k)= & \frac{C_{1}^{2} \bar{\rho}^{2}}{\bar{D}^{2}} b_{g}^{2} \int\left[f_{B}\left(\mathbf{k}_{2}\right)+f_{B}\left(\mathbf{k}_{1}\right)\right] \times f_{B}\left(\mathbf{k}_{2}\right) \\
& \times \frac{P_{\delta}^{\operatorname{lin}}\left(k_{1}\right) P_{\delta}^{\operatorname{lin}}\left(k_{2}\right)}{(2 \pi)^{3}} d^{3} \mathbf{k}_{1} .
\end{aligned}
$$

Note that the $B$-mode intrinsic alignment power spectrum contains no $O\left(P_{\delta}^{\operatorname{lin}}\right)$ contribution. This is because the tidal quadrupole field is a pure $E$-mode (this is even true in the presence of nonlinear evolution), and produces only an $E$-mode pattern of intrinsic alignments if a linear model of galaxy ellipticities such as Eq. (13) applies. Galaxy clustering modulates this field according to the galaxy distribution, and thereby transfers some power into $B$-modes.

The cross power of the matter density and weighted shear is then found from Eq. (15)

$$
P_{\delta, \tilde{\gamma}^{l}}(k)=-\frac{C_{1} \bar{\rho}}{\bar{D}} P_{\delta}^{\operatorname{lin}}(k)
$$

\section{B. Quadratic-alignment model}

The apparent ellipticity of a spiral galaxy is determined principally by the orientation (and hence angular momentum) of its disk. This angular momentum is believed to comes from external tidal fields perturbing the collapsing galaxy to form an anisotropic moment of inertia, which allows the galaxy to be "spun up" by a tidal quadrupole. In this case, the angular momentum vector $\mathbf{L}$ of the galaxy acquires an anisotropic probability distribution, and leads to a mean intrinsic ellipticity. This mean ellipticity vanishes to first order in the tidal field since a tidal field is required both to produce the anisotropic moment of inertia and then to apply a torque. The second-order contribution is [30]

$$
\gamma^{I}=C_{2}\left(T_{x \mu}^{2}-T_{y \mu}^{2}, 2 T_{x \mu} T_{y \mu}\right),
$$

where the tidal tensor is 


$$
T_{\mu \nu}=\frac{1}{4 \pi G}\left(\nabla_{\mu} \nabla_{\nu}-\frac{1}{3} \delta_{\mu \nu} \nabla^{2}\right) S\left[\Psi_{P}\right]
$$

(Since the tidal shears $T$ are spin 2, we recall from the theory of addition of angular momenta that Eq. (19) is the most general quadratic function of $T$ with spin 2.) The resulting density-weighted intrinsic alignment is

$$
\begin{aligned}
\tilde{\gamma}_{E}^{I}(\mathbf{k})= & \frac{C_{2} \bar{\rho}}{(2 \pi)^{3} \bar{D}} \int h_{E}\left(\hat{\mathbf{k}}_{1}^{\prime}, \hat{\mathbf{k}}_{2}^{\prime}\right) \delta_{\operatorname{lin}}\left(\mathbf{k}_{1}^{\prime}\right) \delta_{\operatorname{lin}}\left(\mathbf{k}_{2}^{\prime}\right) \\
& \times\left[\delta^{(3)}\left(\mathbf{k}_{3}^{\prime}\right)+\frac{b_{g}}{(2 \pi)^{3}} \delta_{\operatorname{lin}}\left(\mathbf{k}_{3}^{\prime}\right)\right] d^{3} \mathbf{k}_{1}^{\prime} d^{3} \mathbf{k}_{2}^{\prime},
\end{aligned}
$$

where we have retained the convention that $\mathbf{k}$ is chosen to lie on the $x$ axis, set $\hat{\mathbf{k}}_{a}=\mathbf{k}_{a} /\left|k_{a}\right|$ and $\mathbf{k}_{3}^{\prime}=\mathbf{k}-\mathbf{k}_{1}^{\prime}-$ $\mathbf{k}_{2}^{\prime}$, and defined the $h$-function by $h_{E}=h_{x x}-h_{y y}$ with

$$
h_{\lambda \mu}(\hat{\mathbf{u}}, \hat{\mathbf{v}})=\left(\hat{u}_{\mu} \hat{u}_{\nu}-\frac{1}{3} \delta_{\mu \nu}\right)\left(\hat{v}_{\lambda} \hat{v}_{\nu}-\frac{1}{3} \delta_{\lambda \nu}\right) .
$$

The $B$-mode intrinsic alignment is obtained by replacing $h_{E}$ with $h_{B}=2 h_{x y}$. (See Ref. [49] for a similar calculation without the density weighting $1+\delta_{g}$.)

The predicted intrinsic alignment power spectra $P_{\tilde{\gamma}^{I}}(k)$ from this model can be derived by the same methods as used in Sec. III A, although since $\gamma^{I}$ is second-order in $\Psi_{P}$ (and hence $\delta_{\text {lin }}$ ) there are more Feynman diagrams that contribute. The result is

$$
\begin{aligned}
P_{\tilde{\gamma}^{\prime}}^{E E}(k)= & \frac{C_{2}^{2} \bar{\rho}^{2}}{\bar{D}^{2}}\left\{2 \int\left[h_{E}\left(\hat{\mathbf{k}}_{1}, \hat{\mathbf{k}}_{2}\right)\right]^{2} \frac{P_{\delta}^{\operatorname{lin}}\left(k_{1}\right) P_{\delta}^{\operatorname{lin}}\left(k_{2}\right)}{(2 \pi)^{3}} d^{3} \mathbf{k}_{1}\right. \\
& +\frac{2}{3} b_{g}^{2} \int\left[h_{E}\left(\hat{\mathbf{k}}_{1}^{\prime}, \hat{\mathbf{k}}_{2}^{\prime}\right)+h_{E}\left(\hat{\mathbf{k}}_{2}^{\prime}, \hat{\mathbf{k}}_{3}^{\prime}\right)\right. \\
& \left.+h_{E}\left(\hat{\mathbf{k}}_{3}^{\prime}, \hat{\mathbf{k}}_{1}^{\prime}\right)\right]^{2} \\
& \left.\times \frac{P_{\delta}^{\operatorname{lin}}\left(k_{1}^{\prime}\right) P_{\delta}^{\operatorname{lin}}\left(k_{2}^{\prime}\right) P_{\delta}^{\operatorname{lin}}\left(k_{3}^{\prime}\right)}{(2 \pi)^{6}} d^{3} \mathbf{k}_{1}^{\prime} d^{3} \mathbf{k}_{2}^{\prime}\right\} .
\end{aligned}
$$

In Eq. (23) we have defined $\mathbf{k}_{2}=\mathbf{k}-\mathbf{k}_{1}$ and $\mathbf{k}_{3}^{\prime}=\mathbf{k}-\mathbf{k}_{1}^{\prime}-\mathbf{k}_{2}^{\prime}$.

In the simple version of the quadratic alignment model presented here, there is no density-shear correlation, i.e. $\delta_{\text {lin }}$ and $\left(1+\delta_{g}\right) \gamma^{I}$ have zero cross correlation assuming Gaussian $\delta_{\text {lin }}$, linear biasing $\delta_{g}=b_{g} \delta_{\text {lin }}$, and linear evolution of the density field. We can see this as follows: since the three-point functions of $\delta_{\text {lin }}$ vanish, this correlation is equal to that of $\delta_{\text {lin }}$ and $\delta_{g} \gamma^{I}$. Of the contributing diagrams, those connecting $\delta_{\text {lin }}$ to the $\delta_{g}$ vanish because $\left\langle\gamma^{I}\right\rangle=0$, hence we only consider the diagrams connecting $\delta_{\text {lin }}$ to one of the two tidal quadrupoles $T_{\mu \nu}$ that make up $\gamma^{I}$. But these vanish by symmetry since they must connect $\delta_{g}$, a scalar, to another tidal quadrupole $T_{\mu \nu}$ evaluated at the same point. This fact was first noted in the context of intrinsic alignment contamination of galaxy-galaxy lensing by Ref. [48], which also pointed out that there can be nonzero cross power $P_{\delta, \tilde{\gamma}^{I}}(k)$ if we consider nonlinear density evolution. We will not consider these more complicated models here.

\section{Normalization}

We have normalized the intrinsic alignment amplitude $C_{1}$ for the linear alignment model to match the shear autopower observed by Ref. [33] in the SuperCOSMOS data [50]. Ref. [33] gives the ellipticity variance $\sigma_{e}^{2}(\theta)$ in square cells of side length $\theta$ instead of the shear power spectra; these are related by

$$
\sigma_{e}^{2}(\theta)=\mathcal{R}^{2} \int j_{0}^{2}\left(\frac{\ell_{x} \theta}{2}\right) j_{0}^{2}\left(\frac{\ell_{y} \theta}{2}\right)\left(C_{\ell}^{E E}+C_{\ell}^{B B}\right) \frac{d^{2} \boldsymbol{l}}{4 \pi^{2}},
$$

where $j_{0}(u)=\sin u / u$ and $\mathcal{R}=d e / d \gamma \approx 2$ is the shearto-ellipticity conversion factor. We have assumed a parameterized redshift distribution of the form

$$
\frac{d n}{d z} \propto z^{2} e^{-\left(1.4 z / z_{m}\right)^{1.5}},
$$

where the median redshift for SuperCOSMOS is $z_{m}=$ 0.1 [33]. The SuperCOSMOS ellipticity variances are an order of magnitude greater than the weak lensing prediction and hence must be dominated by intrinsic alignments and/or observational systematics; we have assumed here that the intrinsic alignments dominate, which provides an upper limit if observational systematics are not negligible. We have normalized to the largest scale observation given by Ref. [33], where Eq. (13) is best motivated, and used the ellipticity covariance between the $b_{J}$ and $R$ bandpasses as this is less sensitive to systematics than the variances; this yields $\sigma_{e}^{2}=1.6 \times 10^{-5}$ at $\theta=93.25$ arc min.

For the linear intrinsic alignment model there is a discrete degeneracy in $C_{1}$ since the sign cannot be determined from the ellipticity variance alone; we have used in the plots the positive normalization $C_{1}>0$ because of its physical motivation (stretching of the galaxy along the tidal field).

\section{Results}

In Fig. 2, we have shown the estimated intrinsicalignment contamination of the shear power spectrum for the linear alignment model. Panels 2(a)-2(c)show the contributions to the $E$-mode shear power spectrum assuming a source redshift distribution given by Eq. (25) with median redshifts $z_{m}=0.1,0.5$, and 1.0. In panels 2(d)-2(f)we have shown the intrinsic alignment contributions to the cross spectra between different redshift bins; only the gravitational-intrinsic correlation is nonzero since the redshift distributions do not overlap. For these panels we take the redshift distributions to be Gaussian with $\sigma_{z}=0.1$ (which is much less than the separation of the bins). The contamination $C_{\ell}^{G I}$ is only weakly dependent on $\sigma_{z}$ because the integral in Eq. (11) receives a contribution from a range of radii $\Delta \chi \propto \sigma_{z}$, 
Shear power spectra for linear alignment model

(a) Power spectrum $\left(z_{m}=0.1\right)$

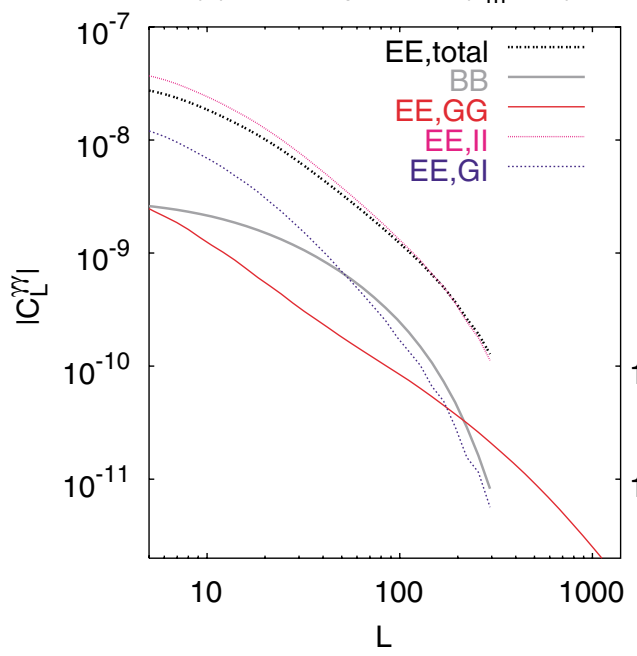

(d) Cross-power $(z=0.5,1.0)$

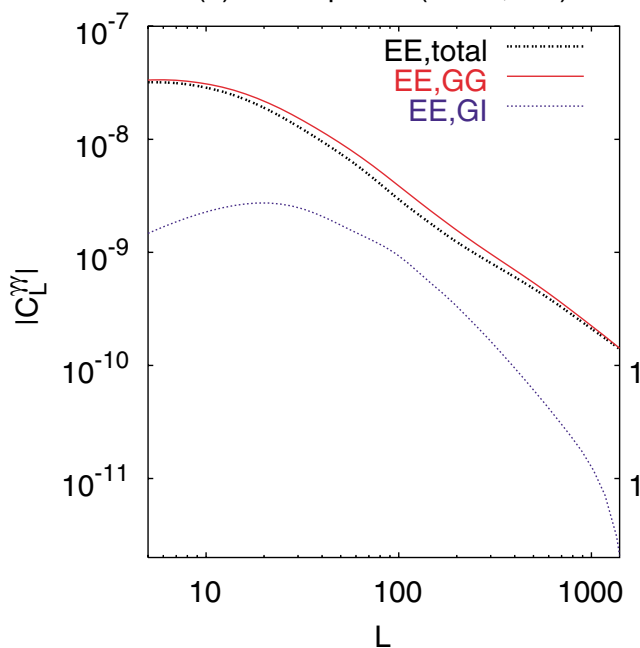

(b) Power spectrum $\left(z_{m}=0.5\right)$

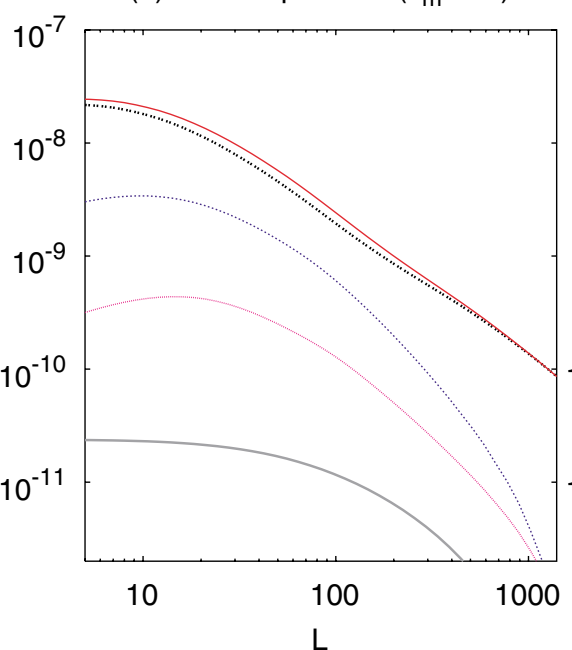

(e) Cross-power $(z=0.5,2.0)$

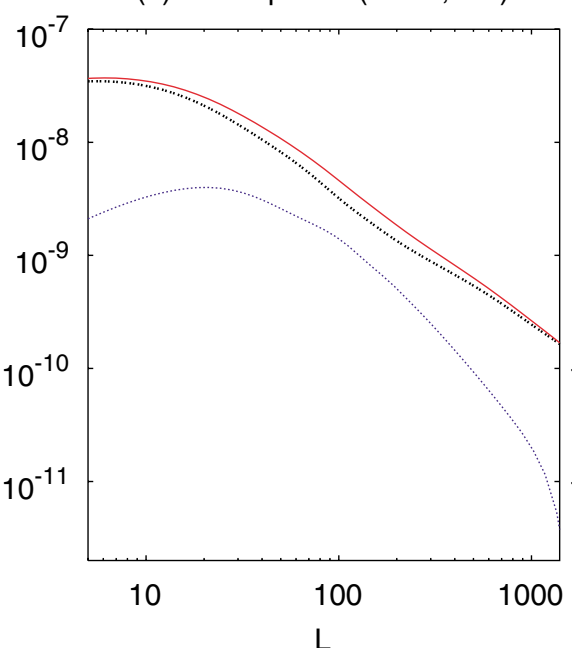

(c) Power spectrum $\left(z_{m}=1.0\right)$

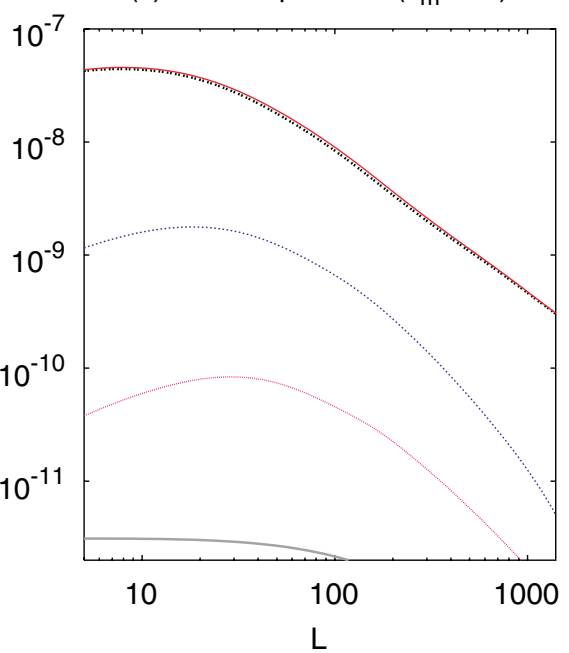

(f) Cross-power ( $\mathrm{z}=1.0,2.0)$

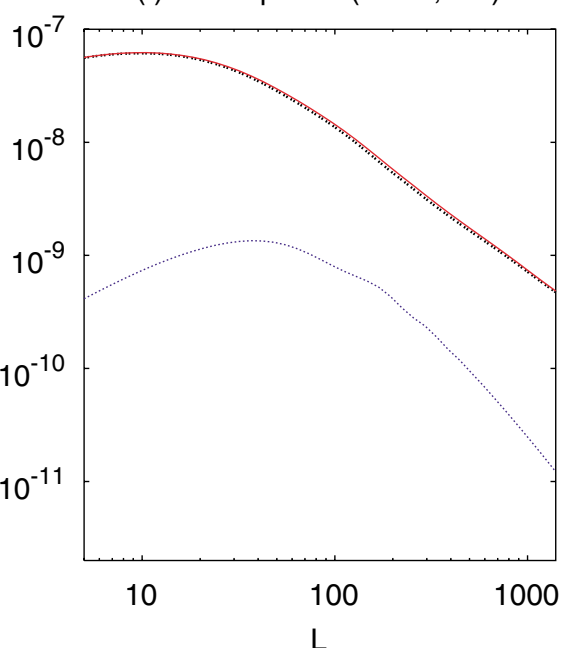

FIG. 2 (color online). The gravitational $(G G)$, intrinsic alignment $(I I)$, and gravitational-intrinsic correlation $(G I)$ contributions to the shear power spectrum, for the linear alignment model. The $B$-mode shear power spectrum is labeled " $\mathrm{B}$ " and has only an intrinsic-intrinsic contribution. The cross term $(G I)$ is negative for this model, so we have plotted its absolute values on the log scale. We show results for (a) the shear power spectrum for a survey with redshift distribution of Eq. (25) with $z_{m}=0.1$; (b) and (c) similar for $z_{m}=0.5$ and 1.0, respectively; (d) the shear cross-power between redshifts $z=0.5$ and $z=1.0$ (the slices have Gaussian redshift distributions with width $\sigma_{z}=0.1$ ); (e) the shear cross-power between redshifts $z=0.5$ and $z=2.0$; and (f) the shear cross-power between redshifts $z=1.0$ and $z=2.0$. The intrinsic alignment amplitude $C_{1}$ is normalized to SuperCOSMOS. Panel (a) is cut off at $\ell=300$, roughly the smoothing scale used for the intrinsic alignment calculation, since the model does not make sense on smaller scales.

whereas the integrand contains a factor of $f_{\beta}(\chi)$, whose peak value scales $\propto \sigma_{z}^{-1}$.

The most striking result from Fig. 2 is that the gravitational-intrinsic contribution dominates the contamination of the shear power spectrum for the broad redshift distribution [Fig. 2(c). Even if the intrinsicintrinsic shear correlations are small (in this case they are $0.5 \%$ of the lensing signal for the broad redshift distribution with $z_{m}=1.0$ at $\ell=500$ ), the gravitational- intrinsic contamination to the cross spectra can still be large $(\sim 5 \%$ at $\ell=500)$. This contamination is especially pronounced for the cross spectrum between the widely separated bins at $z=0.5$ and 2.0 [Fig. 2(e): here $\left|C_{\ell}^{G I} / C_{\ell}^{G G}\right|$ can be as large as $\sim 30 \%$.

As noted above, there is no correlation between the gravitational and intrinsic shears in the quadratic alignment model. Thus there is only intrinsic alignment contamination if the redshift distributions overlap, and the 
contamination is much lower because it scales as the square of the intrinsic alignment amplitude $\propto C_{2}^{2}$ instead of linearly as in the case of the GI cross correlation.

\section{METHODS TO ASSESS THE CONTAMINATION}

So far we have considered the contamination of the power spectra in cosmic shear surveys due to intrinsic alignments, and shown that the interference between gravitational and intrinsic shears can dominate the contamination and reach levels that are important for high precision studies of weak lensing. In this section we discuss two methods to estimate the amplitude of the effect and separate it from the weak lensing effect. The first method is based on density-shear correlations, while the second method is purely geometrical and uses only scaling of the cross correlation signal with redshift.

\section{A. Density-shear cor relations}

We are assuming there is a correlation between the intrinsic alignment and gravitational potential field that gives rise to shear: the latter can be reconstructed from the density field. On large scales we can assume galaxy density field is linearly proportional to the density field up to a constant, the so called bias parameter. In fact, there is no need to do the reconstruction itself: at the two-point function level the full information on this correlation is encoded in the density-shear correlation. We can thus constrain the intrinsic alignment models by comparing them to the observed density-shear correlation from galaxy surveys.

To date these measurements have been done on small scales $<1 h^{-1} \mathrm{Mpc}$ for the purpose of understanding contamination of galaxy-galaxy lensing. The quantity measured in these studies is $\Delta \gamma(r)$, defined as the mean tangential shear of a "satellite" galaxy a transverse distance $r$ from the "primary." This is equal to

$$
\Delta \gamma(r)=\frac{\int\left\langle\left[1+\delta_{g}(0)\right]\left[1+\delta_{g}(x)\right] \gamma_{+}^{I}(x)\right\rangle d x_{\|}}{\int\left\langle\left[1+\delta_{g}(0)\right] \delta_{g}(x)\right\rangle d x_{\|}},
$$

cf. Eq. (A5) of Ref. [51]. The idea behind this equation is that we compute the galaxy-shear correlations for all close angular pairs on the sky and then use the corresponding galaxy-galaxy correlation to estimate what fraction of these pairs is physically close also in threedimensional space. The signal is then boosted by dividing by this fraction.

Noting that $\left(1+\delta_{g}\right) \gamma_{+}^{I}=\tilde{\gamma}_{+}^{I}$, and taking the Fourier transform in the transverse dimensions, we get

$$
\Delta \gamma(r)=\frac{\int P_{\delta_{g}, \tilde{\gamma}^{I}}(k) J_{2}(k r) k d k}{\int P_{\delta_{g}}(k) J_{0}(k r) k d k}=\frac{\int P_{\delta, \tilde{\gamma}^{I}}(k) J_{2}(k r) k d k}{b_{g} \int P_{\delta}(k) J_{0}(k r) k d k}
$$

In Fig. 3 we have plotted $\Delta \gamma(r)$ as measured in the Sloan Digital Sky Survey (SDSS) [51]; the sample was dominated by objects at $z \approx 0.1$ and so we have displayed

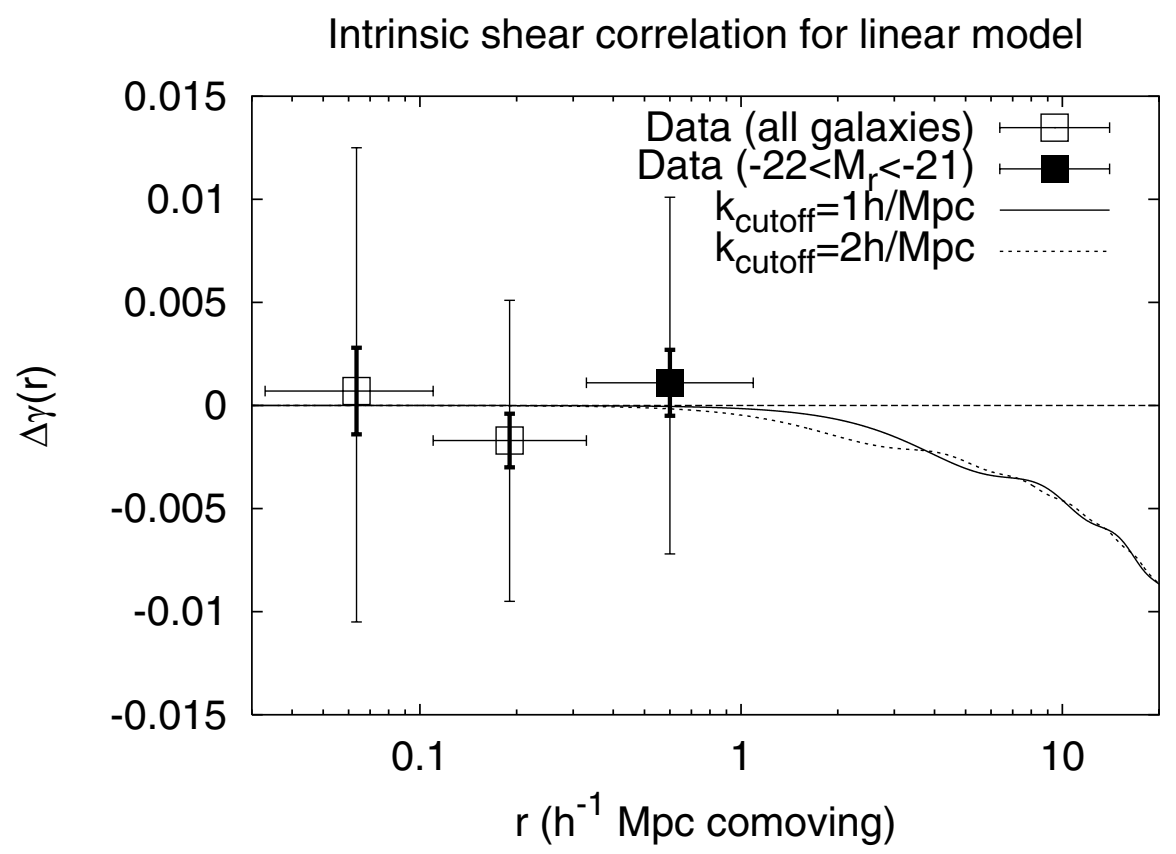

FIG. 3. The intrinsic shear statistic $\Delta \gamma(r)$ of Eq. (26). The SDSS data points are shown, with the horizontal error bars indicating the range of radii used, the thick vertical error bars indicating $1 \sigma$ statistical errors, and the thin vertical error bars representing 99.9\% confidence limits including systematics (principally shear calibration and removal of lensing signal) [51]. 
the model prediction at that redshift. (The data points were presented in Ref. [51] in physical separation units; we have converted them to comoving separation at $z=0.1$.)

We see from Fig. 3 that the model predicts a constant value of density-shear correlations on large scales, as expected in a linear bias model. On scales smaller than the initial size of a galactic halo one does not expect much correlation. The transition scale is rather uncertain and depends on the somewhat arbitrary value of the cutoff we used in the calculation. A typical value should be given by the scale length within which the enclosed mass is of the order of a typical galactic halo mass, of order $10^{12} M_{\odot}$. This gives $k_{\text {cutoff }} \sim(1-2) h / \mathrm{Mpc}$, which are the two values used in Fig. 3. We see that the current constraints are inconclusive because they do not extend to sufficiently large scales. The results from this figure also suggest that galaxy-shear correlations are a powerful probe of identifying this contamination and that there is plenty of statistical power in the SDSS data to estimate the contamination by extending the analysis of Ref. [51] to larger transverse separations.

\section{B. Geometric projection}

The analysis of intrinsic alignment contamination should not be based solely on low-redshift information: the local universe may not accurately reflect the intrinsic alignments of the weak lensing source galaxies since (for example) mergers could cause the intrinsic alignment signal to evolve with redshift. Consistency of shear cross spectra obtained from different types of galaxies in the same range of redshifts would argue against significant gravitational-intrinsic correlations, but if an inconsistency is observed that is not attributable to observational systematics, then some method of separating pure lensing $(G G)$ from gravitational-intrinsic $(G I)$ signals will be needed.

One possibility is to take advantage of the different redshift dependence in the $G G$ and $G I$ signals when applied to tomography between slices at redshifts $z_{\alpha}<$ $z_{\beta}$ (the redshift slices should be separated far enough to eliminate overlaps in their distribution and hence $I I$ contamination). In the idealized case where the photo- $z$ s for the nearby redshift bin $z_{\alpha}$ have negligible uncertainty, the $G I$ signal at a fixed $\ell$ and $z_{\alpha}$ rises with increasing $z_{\beta}$ as

$$
C_{\ell}^{E E, G I}\left(z_{\alpha}, \beta\right) \propto \int \frac{D_{A}\left(z_{\alpha}, z\right)}{D_{A}(0, z)}[1+\zeta(z)]\left(\frac{d n}{d z}\right)_{\beta} d z,
$$

where $D_{A}$ is the comoving angular diameter distance separating two redshifts and $\zeta(z)$ is the shear calibration error for galaxies at redshift $z$ (which ideally would be made negligible). The $G G$ (pure lensing) signal also rises with increasing $z_{\beta}$, but more slowly because $z_{\alpha}$ in Eq. (28) is replaced by the redshift of the contributing structures, which must be $<z_{\alpha}$. In principle, one could project out signals in tomography that have the redshift dependence of Eq. (28).

If the effective ratio of angular diameter distances $g_{\alpha \beta}$ is measured by cosmography-i.e., measurement of the relative shear signal at different $z_{\beta}$ behind a sample of lenses at $z_{\alpha}$-then provided that the same source sample at $z_{\beta}$ is used for tomography as for cosmography, the right-hand side of Eq. (28) is obtained directly [52,53] up to an irrelevant multiplicative factor. This geometrical method has the practical advantage of automatically accounting for any shear calibration errors, even if they are redshift-dependent, and eliminating the need for external information about the angular diameter distance function $D_{A}$. Since the density-intrinsic shear correlation may evolve with redshift, the deprojection of signals proportional to Eq. (28) will have to be done separately for each redshift slice $z_{\alpha}$. The method is more accurate if the slices are narrow so that the effective $z_{\alpha}$ for the intrinsic alignment does not differ significantly from the effective $z_{\alpha}$ for cosmography, thus precise photo-zs would be extremely valuable.

The geometric method is model-independent in the sense that it works for arbitrary $k$ and $z$-dependence of the density-intrinsic shear correlation, but of course some cosmological information is lost. An alternative approach would be to marginalize over a parametrization of $P_{\delta, \tilde{\gamma}^{I}}(k, z)$ (perhaps forced to match on to the $P_{\delta, \tilde{\gamma}^{I}}(k)$ measured in the local universe at $z \approx 0$ ); this may retain more cosmological information if $P_{\delta, \tilde{\gamma}^{I}}(k, z)$ is, e.g., forced to be a smooth function of $z$, but of course it sacrifices the model independence of the geometric technique.

\section{DISCUSSION}

In this paper, we have considered the contamination of the power spectrum in cosmic shear surveys due to intrinsic alignments, and shown that the gravitational and intrinsic shears need not be independent. For some models, the contamination is dominated by the gravitationalintrinsic cross power rather than the power spectrum of the intrinsic alignments themselves. One implication of this is that the $B$-mode signal, frequently used as a systematics test, may be a misleading indicator of intrinsic alignment contamination, since it is sensitive only to the intrinsic-intrinsic autopower $C_{\ell}^{B B, I I}$ but not to the gravitational-intrinsic cross-power $C_{\ell}^{G I}$, which can only be nonvanishing for the $E$ modes. Figure 2(c) provides an extreme example: here the change in $C_{\ell}^{E E}$ due to intrinsic alignments exceeds the $B$-mode power spectrum $C_{\ell}^{B B}$ by $>2$ orders of magnitude. The opposite extreme is the quadratic-alignment model in which there is no gravitational-intrinsic correlation and the $E$ and $B$ 
mode contributions from intrinsic alignments are similar.

A realistic model of intrinsic alignments will likely fall somewhere in between these two extremes. The linear alignment model [Eq. (13)] obviously predicts maximal correlation between the density and intrinsic shear fields on large scales in the sense that the correlation coefficient $\rho_{\delta, \tilde{\gamma}^{I}}(k)=P_{\delta, \tilde{\gamma}^{I}}(k) / \sqrt{P_{\delta}(k) P_{\tilde{\gamma}^{I}}(k)}$ has absolute value $\approx 1$. Any higher-order corrections to Eq. (13) will therefore reduce the correlation coefficient. On the other hand, the simplest form of the quadratic-alignment model [Eq. (19)] is probably too optimistic, even though the model is well-motivated by tidal torque theory. We found $P_{\delta, \tilde{\gamma}^{I}}(k)=0$ for this model not because of any fundamental symmetry principle, but rather because the assumed Gaussianity of the density field causes third-order statistics in $\delta$ to vanish. In the real universe we can expect tidally torqued galaxies to have $P_{\delta, \tilde{\gamma}^{I}}(k) \neq 0$ because of non-Gaussianity from nonlinear evolution [48], and because of third- and higher-order corrections to Eq. (19) that we have neglected.

While we have presented these results for the power spectrum, the non-Gaussianity of the density field, and hence of cosmic shear, has motivated studies of other statistics, most notably the bispectrum [54-59]. The bispectrum can in principle pick up three types of intrinsic alignment contamination: the intrinsic shear bispectrum (III), and the gravitational-gravitational-intrinsic $(G G I)$ and gravitational-intrinsic-intrinsic $(G I I)$ cross bispectra. If tomography is used, the measured bispectrum components are $B_{\ell_{\alpha} \ell_{\beta} \ell_{\gamma}}^{E E E}(\alpha \beta \gamma)$, where $\alpha, \beta$, and $\gamma$ are indices indicating the source bins centered at redshifts $z_{\alpha} \leq$ $z_{\beta} \leq z_{\gamma}$. Assuming that these bins do not overlap significantly in redshift, the III contaminant is only nonvanishing for the triplets where all three source galaxies lie in the same redshift slice $z_{\alpha}=z_{\beta}=z_{\gamma}$; this is only a small fraction of the triplets and hence little statistical power is lost by rejecting them [37]. GGI is produced by a density-density-intrinsic shear bispectrum in the nearest of the three slices, and it can in principle contaminate any tomographic shear bispectrum in which $z_{\alpha}<z_{\beta} \leq z_{\gamma}$. GII is produced by the density-intrinsic-intrinsic bispectrum, again in the nearest of the three slices, but this time in order to have the intrinsic shear be correlated and to produce a lensing effect on the more distant slice, GII satisfies a "selection rule" $z_{\alpha}=z_{\beta}<z_{\gamma}$. Thus every triplet of source screens is potentially contaminated by exactly one of GGI, GII, or III. Note also that the quadratic-alignment model (Sec. III B), which at lowest order predicted no GI contamination to the power spectrum, does predict a $G G I$ contamination $B_{\delta, \delta, \tilde{\gamma}^{I}}\left(k_{1}, k_{2}, k_{3}\right) \neq 0$ because the intrinsic shear $\gamma^{I}$ is a quadratic function of $\delta$. We defer a calculation of the magnitude of this effect to a future paper.
Our analysis clearly motivates observations to constrain the density-intrinsic shear correlation. Just as the intrinsic shear autopower can be constrained by lowredshift measurements where the lensing signal is negligible $[28,33]$, so it should be possible to use these same low-redshift measurements to measure $P_{\delta, \tilde{\gamma}^{I}}(k)$ provided that a tracer of the density field is available. The number density of galaxies is one possibility; recent observations have probed the galaxy-intrinsic shear correlation on $0.02-1 h^{-1} \mathrm{Mpc}$ scales [51,60], and it would be useful to extend these studies to larger scales where linear biasing is valid. (At least one density-shear correlation measurement is available on large scales [29]. These authors were interested primarily in using intrinsic alignments to reconstruct the density field; they therefore did not use lensing shear estimators, and they measured threedimensional separations in redshift space, so their results are difficult to interpret in the present context.) For such observations, it would also be useful to distinguish types of galaxies (e.g. early vs late); if one type of galaxy turns out to have significantly greater $P_{\delta, \tilde{\gamma}^{I}}(k)$, the shear power spectra could be performed principally on the other types so as to reduce contamination.

A second approach to eliminating the contamination is to project out the information that correlates with the known redshift scaling of the signal in the cross correlations between narrow redshift bins, assuming these are available from the photo- $z$ methods. This method is geometric and achieves perfect decontamination under these idealized assumptions, but projection does destroy some information in the data. It also puts severe requirements on the accuracy of photometric redshifts, which may require many passbands to achieve this [61].

In summary, we have shown that cosmic shear surveys will need to consider the possibility of intrinsic alignment contamination from its interference with weak lensing-induced shear. The magnitude of the effect is extremely model-dependent and can range from essentially zero (simplest quadratic alignment models) to severe (simplest linear alignment models). In the latter case it can exceed intrinsic correlations by an order of magnitude and cannot be identified using $B$-mode power spectrum, nor can it be eliminated using cross correlations between different redshift slices. This result is disappointing and implies that this effect could be very damaging for efforts to use weak lensing as a high precision test of cosmology with future surveys such as PanSTARRS, LSST or SNAP. However, the situation is far from hopeless: here we have considered several possible ways to constrain and/or suppress the contamination. The next step observationally is a density-shear correlation analysis with the shear computed using a lensing estimator in a wide-angle, shallow survey such as the Sloan Digital Sky Survey [62] to constrain $P_{\delta, \tilde{\gamma}^{I}}(k, z \ll 1)$; the next step theoretically is a thorough analysis of the vari- 
ous methods described above for suppressing the gravitational-intrinsic contamination, in particular, to understand how much degradation on the cosmological parameters occurs and whether they increase sensitivity to any of the other potential systematics such as photo- $z$ errors, shear calibration errors, and spurious power.

\section{ACKNOWLEDGMENTS}

C. H. acknowledges the support of the NASA Graduate Student Researchers Program (GSRP). U.S. is supported by Packard Foundation, NASA NAG5-11489 and NSF CAREER-0132953.
[1] L. Van Waerbeke, Y. Mellier, T. Erben, J. C. Cuillandre, F. Bernardeau, R. Maoli, E. Bertin, H. J. Mc Cracken, O. Le Fèvre, and B. Fort et al., Astron. Astrophys. 358, 30 (2000).

[2] D. J. Bacon, A. R. Refregier, and R. S. Ellis, Mon. Not. R. Astron. Soc. 318, 625 (2000).

[3] J. Rhodes, A. Refregier, and E. J. Groth, Astrophys. J. Lett. 552, L85 (2001).

[4] H. Hoekstra, H. K. C. Yee, M. D. Gladders, L. F. Barrientos, P. B. Hall, and L. Infante, Astrophys. J. 572, 55 (2002).

[5] M. Jarvis, G. M. Bernstein, P. Fischer, D. Smith, B. Jain, J. A. Tyson, and D. Wittman, Astron. J. 125, 1014 (2003).

[6] L. Van Waerbeke, Y. Mellier, R. Pelló, U.-L. Pen, H. J. McCracken, and B. Jain, Astron. Astrophys. 393, 369 (2002).

[7] M. L. Brown, A. N. Taylor, D. J. Bacon, M. E. Gray, S. Dye, K. Meisenheimer, and C. Wolf, Mon. Not. R. Astron. Soc. 341, 100 (2003).

[8] R. Massey, A. Refregier, D. Bacon, and R. Ellis, astro-ph/ 0404195

[9] http://www.cfht.hawaii.edu/Science/CFHLS/

[10] http://pan-starrs.ifa.hawaii.edu/public/

[11] http://snap.lbl.gov/

[12] http://www.lsst.org/

[13] W. Hu and M. Tegmark, Astrophys. J. Lett. 514, L65 (1999).

[14] D. J. Eisenstein, W. Hu, and M. Tegmark, Astrophys. J. 518, 2 (1999)

[15] M. Zaldarriaga, D. N. Spergel, and U. Seljak, Astrophys. J. 488, 1 (1997).

[16] J. R. Bond, G. Efstathiou, and M. Tegmark, Mon. Not. R. Astron. Soc. 291, L33 (1997).

[17] W. Hu, Phys. Rev. D 65, 023003 (2002).

[18] W. Hu, Phys. Rev. D 66, 083515 (2002).

[19] N. Kaiser, Astrophys. J. 537, 555 (2000).

[20] T. Erben, L. Van Waerbeke, E. Bertin, Y. Mellier, and P. Schneider, Astron. Astrophys. 366, 717 (2001).

[21] D. J. Bacon, A. Refregier, D. Clowe, and R. S. Ellis, Mon. Not. R. Astron. Soc. 325, 1065 (2001).

[22] G. M. Bernstein and M. Jarvis, Astron. J.123, 583 (2002).

[23] L. Van Waerbeke and Y. Mellier, astro-ph/0305089.

[24] C. Hirata and U. Seljak, Mon. Not. R. Astron. Soc. 343, 459 (2003).

[25] R. A. C. Croft and C. A. Metzler, Astrophys. J. 545, 561 (2000).

[26] A. Heavens, A. Refregier, and C. Heymans, Mon. Not. R. Astron. Soc. 319, 649 (2000).
[27] J. Lee and U. Pen, Astrophys. J. Lett. 532, L5 (2000).

[28] U. Pen, J. Lee, and U. Seljak, Astrophys. J. Lett. 543, L107 (2000).

[29] J. Lee and U. Pen, Astrophys. J. 555, 106 (2001).

[30] P. Catelan, M. Kamionkowski, and R. D. Blandford, Mon. Not. R. Astron. Soc. 320, L7 (2001).

[31] R. G. Crittenden, P. Natarajan, U. Pen, and T. Theuns, Astrophys. J. 559, 552 (2001).

[32] Y. P. Jing, Mon. Not. R. Astron. Soc. 335, L89 (2002).

[33] M. L. Brown, A. N. Taylor, N. C. Hambly, and S. Dye, Mon. Not. R. Astron. Soc. 333, 501 (2002).

[34] J. Lee and U. Pen, Astrophys. J. Lett. 567, L111 (2002).

[35] L. King and P. Schneider, Astron. Astrophys. 396, 411 (2002).

[36] C. Heymans and A. Heavens, Mon. Not. R. Astron. Soc. 339, 711 (2003).

[37] M. Takada and M. White, Astrophys. J. Lett. 601, L1 (2004).

[38] L. J. King and P. Schneider, Astron. Astrophys. 398, 23 (2003).

[39] C. Heymans, M. Brown, A. Heavens, K. Meisenheimer, A. Taylor, and C. Wolf, Mon. Not. R. Astron. Soc. 347, 895 (2004).

[40] W. Hu and T. Okamoto, Astrophys. J. 574, 566 (2002).

[41] C. M. Hirata and U. Seljak, Phys. Rev. D 67, 43001 (2003).

[42] P. Schneider, L. van Waerbeke, and Y. Mellier, Astron. Astrophys. 389, 729 (2002).

[43] N. Kaiser, Astrophys. J. 388, 272 (1992).

[44] M. Tegmark, M. A. Strauss, M. R. Blanton, K. Abazajian, S. Dodelson, H. Sandvik, X. Wang, D. H. Weinberg, I. Zehavi, N. A. Bahcall et al., Phys. Rev. D 69, 103501 (2004).

[45] D. J. Eisenstein and W. Hu, Astrophys. J. 496, 605 (1998).

[46] J. A. Peacock and S. J. Dodds, Mon. Not. R. Astron. Soc. 280, L19 (1996).

[47] F. C. van den Bosch, T. Abel, R. A. C. Croft, L. Hernquist, and S. D. M. White, Astrophys. J. 576, 21 (2002).

[48] L. Hui and J. Zhang, astro-ph/0205512.

[49] J. Mackey, M. White, and M. Kamionkowski, Mon. Not. R. Astron. Soc. 332, 788 (2002).

[50] N.C. Hambly, H.T. MacGillivray, M. A. Read, S. B. Tritton, E. B. Thomson, B. D. Kelly, D. H. Morgan, R. E. Smith, S. P. Driver, J. Williamson et al., Mon. Not. R. Astron. Soc. 326, 1279 (2001).

[51] C. M. Hirata, R. Mandelbaum, U. Seljak, J. Guzik, N. Padmanabhan, C. Blake, J. Brinkmann, T. Budavari, A. 
Connolly, I. Csabai et al., Mon. Not. R. Astron. Soc. 353, 529 (2004).

[52] G. Bernstein and B. Jain, Astrophys. J. 600, 17 (2004).

[53] B. Jain and A. Taylor, Phys. Rev. Lett. 91, 141302 (2003).

[54] A. Cooray and W. Hu, Astrophys. J. 548, 7 (2001).

[55] L. Van Waerbeke, T. Hamana, R. Scoccimarro, S. Colombi, and F. Bernardeau, Mon. Not. R. Astron. Soc. 322, 918 (2001).

[56] T. Hamana, S. T. Colombi, A. Thion, J. E. G. T. Devriendt, Y. Mellier, and F. Bernardeau, Mon. Not. R. Astron. Soc. 330, 365 (2002).

[57] T. Zhang, U. Pen, P. Zhang, and J. Dubinski, Astrophys. J. 598, 818 (2003).
[58] S. Ho and M. White, Astrophys. J. 607, 40 (2004).

[59] M. Takada and B. Jain, Mon. Not. R. Astron. Soc. 348, 897 (2004).

[60] G. M. Bernstein and P. Norberg, Astron. J. 124, 733 (2002).

[61] D. J. Bacon, A. N. Taylor, M. L. Brown, M. E. Gray, C. Wolf, K. Meisenheimer, S. Dye, L. Wisotzki, A. Borch, and M. Kleinheinrich, astro-ph/0403384.

[62] D. G. York, J. Adelman, J. E. Anderson, S. F. Anderson, J. Annis, N. A. Bahcall, J. A. Bakken, R. Barkhouser, S. Bastian, E. Berman et al., Astron. J. 120, 1579 (2000). 